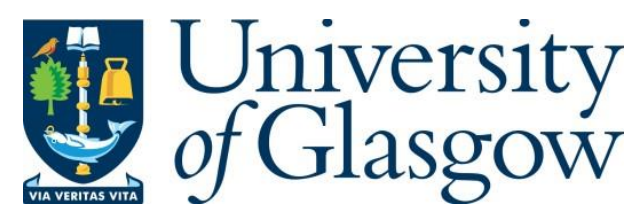

Ahmed, A., Ur Rehman, M. and Abbasi, Q. H. (2018) Miniature Implantable Antenna Design for Blood Glucose Monitoring. In: 2018 International Applied Computational Electromagnetics Society Symposium (ACES), Denver, CO, USA, 25-29 March 2018, ISBN 9780996007870

(doi:10.23919/ROPACES.2018.8364219)

There may be differences between this version and the published version. You are advised to consult the publisher's version if you wish to cite from it.

http://eprints.gla.ac.uk/201475/

Deposited on: 14 November 2019

Enlighten - Research publications by members of the University of Glasgow http://eprints.gla.ac.uk 


\section{Miniature Implantable Antenna Design for Blood Glucose Monitoring}

\author{
$1^{\text {st }}$ Ayesha Ahmed \\ School of Comp. Science \& Tech. \\ University of Bedfordshire \\ Luton LU1 3JU, United Kingdom \\ ayesha.ahmed@study.beds.ac.uk
}

\author{
$2^{\text {nd }}$ Masood Ur-Rehman \\ School of Comp. Science \& Tech. \\ University of Bedfordshire \\ Luton LU1 3JU, United Kingdom \\ masood.urrehman@beds.ac.uk
}

$3^{\text {rd }}$ Qammer Hussain Abbasi
School of Engineering
University of Glasgow

Glasgow G12 8QQ, United Kingdom

qammer.abbasi@glasgow.ac.uk

\begin{abstract}
In this paper, a miniaturised implantable antenna with the dimensions of $8 \times 8 \times 1 \mathrm{~mm}^{3}$ has been studied for continuous monitoring of Blood Glucose Levels (BGL). The antenna performance is analysed numerically for both free space and implanted operation. It has the lowest resonant frequency of $3.58 \mathrm{GHz}$ in free space with a gain $1.18 \mathrm{GHz}$ while operates at $2.58 \mathrm{GHz}$ with a gain of $4.18 \mathrm{dBi}$ when implanted. Good performance and small size make it a good for implantable glucose monitoring devices.
\end{abstract}

Index Terms-Implanatable antennas, Glucose monitoring.

\section{INTRODUCTION}

Diabetes, the most worried threat to the modern people, has brought about substantial economic loss not only to people with them but also to the public health system and national economy system [1]. According to [2], effective diabetes management could reduce the risk of the complications associated with the disease; therefore, real time monitoring techniques are indispensable. Among these techniques, implanted glucose sensors attract a lot of interest [3] and various implanted antenna are designed [4]-[6], where the miniaturization of the antenna with high performances is still a great challenge. To realise such antennas, several methods like high-permittivity substrates, fractal structures, shorting pins loading and etc. have been studied [7]. The high permittivity method and split-ring resonator (SRR) is more commonly used techniques. In [5], the miniaturization was completed realising an antenna size of $8.5 \times 8.5 \times 1.27 \mathrm{~mm}^{3}$ by loading C-shaped slots and Complimentary Split-Ring Resonator (CSRR) on the radiation patch. However, there is a need to further decrease the size of the antenna to meet the requirements of continually miniaturizing implantable devices.

In this paper, following the work in [5], efforts are made to further reduce the antenna size. A smaller implantable antenna size is realised for glucose monitoring located in subcutaneous layer of the human tissues with the techniques of the CSRR and high permittivity substrates. The antenna performance is studied through numerical modelling and analysis. The paper is organized as follows: Section II presents the configuration of the proposed implantable antenna and the numerical model of the human body phantom with antenna injected in the subcutaneous layers.
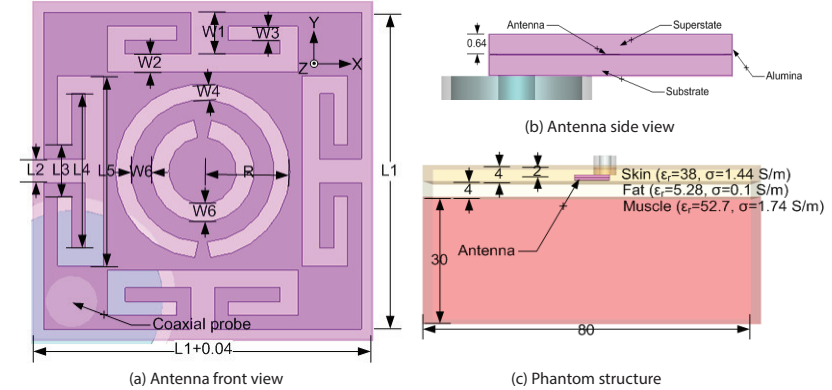

Fig. 1. Schematics of the antenna and three-layer phantom with implanted antenna.

Section III gives the analysis of the antenna performance in terms of numerical results. Conclusions are drawn in Section IV.

\section{Antenna Design AND Discussion}

\section{A. Antenna design}

The structure of the proposed antenna is shown in Fig. 1 (a-b) while the corresponding geometrical parameters are given in Table I. The antenna is modelled and simulated using Computer Simulation Technology (CST) Microwave Studio package. The substrate and superstate used are the lossy Rogers TMM 13i $\left(\epsilon_{r}=12.85, \tan \delta=0.0019\right)$ with a height of $0.5 \mathrm{~mm}$. The patch and ground are all considered as Perfect Electric Conductors (PEC) for simplification having the length and width of same size as the substrate and thickness of $35 \mu \mathrm{m}$. The antenna is fed through a 50 ohm coaxial probe. The SMA connector is also modelled as shown in Fig. 1(b). To alleviate the effects of the human tissues, the antenna is covered in a film made of Alumina with $\epsilon_{r}=9.2, \tan \delta=0.008$ and a thickness of $0.02 \mathrm{~mm}$.

\section{B. Numerical Phantom Model}

The in-body antenna performance is tested using a threelayer numerical phantom of size $80 \times 80 \times 38 \mathrm{~mm}^{3}$ and implanting it $2 \mathrm{~mm}$ in the skin. The phantom consists of three layers, i.e., skin, fat, and muscle. The configuration of the phantom with electrical parameters (the relative permittivity and the conductivity [8]) in the ISM band is shown in Fig. 1(c). 
TABLE I

STRUCTURAL PARAMETERS OF THE PROPOSED ANTENNA

\begin{tabular}{cc|cc}
\hline \hline Ant. Para. & Value $(\mathrm{mm})$ & Ant. Para. & Value $(\mathrm{mm})$ \\
\hline$W_{1}$ & 1.05 & $L_{1}$ & 8.0 \\
\hline$W_{2}$ & 0.45 & $L_{2}$ & 0.6 \\
\hline$W_{3}$ & 0.35 & $L_{3}$ & 1.3 \\
\hline$W_{4}$ & 0.4 & $L_{4}$ & 3.9 \\
\hline$W_{5}$ & 0.5 & $L_{5}$ & 4.8 \\
\hline$W_{6}$ & 0.45 & $R$ & 2.2 \\
\hline \hline
\end{tabular}

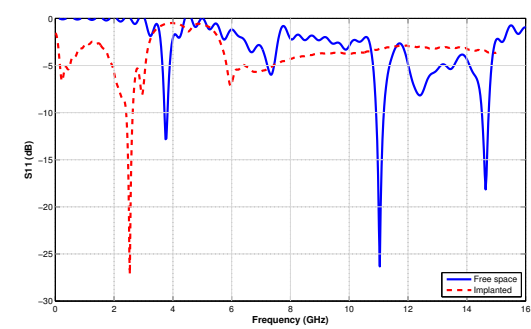

Fig. 2. S11 response of the antenna in free space and implanted configurations.

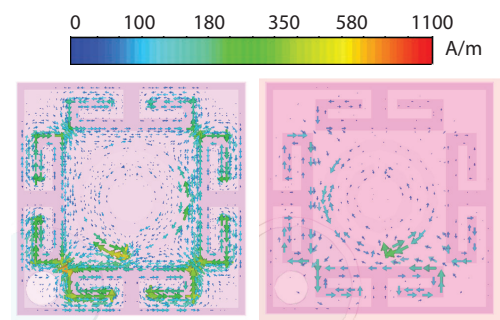

(a) $3.75 \mathrm{GHz}$

(b) $2.58 \mathrm{GHz}$ (implanted)

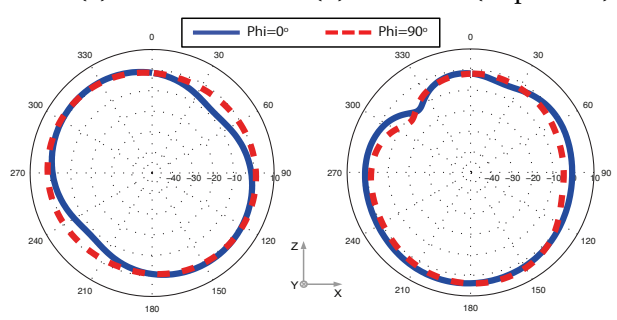

(c) $3.75 \mathrm{GHz}$

(d) $2.58 \mathrm{GHz}$ (implanted)

Fig. 3. Surface current distribution and radiation patterns for proposed antenna's operation in free space at $3.75 \mathrm{GHz}$ and in implantable condition at $2.58 \mathrm{GHz}$.

\section{NUMERICAL RESULTS AND ANALYSIS}

The antenna performance is analysed in terms of surface current distribution, reflection coefficient, radiation pattern and gain in free space and implanted configurations. The reflection coefficient $\left(S_{11}\right)$ response of the proposed antenna in free space and in the phantom are shown in Fig. 2. It can be seen that the resonance frequency changes from $3.75 \mathrm{GHz}$ in free space to $2.58 \mathrm{GHz}$ with excellent impedance matching for the antenna in implanted condition. For the free space case, the antenna has various parasitic resonances in the higher frequency band but it has limited effect on the antenna performance when the antenna is implanted in human body.

Fig. 3(a-b) shows the current distribution on the antenna surface for the two operational conditions at free space frequencies of $3.75 \mathrm{GHz}$ and implanted frequency of 2.58 GHz. It shows that the current path has increased with the introduction of the slots and CSRR in the patch thus lowering the resonant frequency. The radiation patterns are observed at $3.75 \mathrm{GHz}$ for the free space operation while at $2.58 \mathrm{GHz}$ for the implanted scenario as illustrated in Fig. 3(c-d). These results indicate that the antenna has good radiation coverage in both $\phi=0^{\circ}$ and $\phi=90^{\circ}$ planes with nearly omni-directional far-field at all the observed frequencies. More tellingly, the antenna successfully maintains the pattern shape and coverage in both the planes when implanted into the skin tissues as evident from Fig. 3(d). The near-omnidirectionallty helps the antenna to send and receive the signal/information in all directions, hence mitigating possible body posture and shape implications. The simulated peak gain of the proposed antenna in free space is noted to be $1.8 \mathrm{dBi}, 4.0 \mathrm{dBi}$ and $5,4 \mathrm{dBi}$ at 3.75 $\mathrm{GHz}, 11 \mathrm{GHz}$ and $14 \mathrm{GHz}$. In implanted scenario, the gain is observed to be $4.18 \mathrm{dBi}$ at $2.58 \mathrm{GHz}$. It is evident that the proposed antenna offers a good gain performance in all working conditions.

\section{CONCLUSION}

A miniaturized implantable antenna has been studied for the free space and implanted conditions for continuous blood sugar monitoring. The antenna performance is analysed numerically using a three-layer human body phantom. The results have shown that good radiation coverage and a peak gain of $4.18 \mathrm{dBi}$ could be obtained despite the small size of the antenna at $2.58 \mathrm{GHz}$ when implanted in body. Small size, good gain and resilience to the human body effects makes the antenna a good candidate for tele-health monitoring systems.

\section{REFERENCES}

[1] World Health Organization et al. Global report on diabetes. World Health Organization, 2016.

[2] Horizon Scanning Research and Intelligence Centre. New and emerging non-invasive glucose monitoring. Horizon Scanning Research and Intelligence Centre, 2016.

[3] P. S. Hall and Y. Hao. Antennas and Propagation for Body-Centric Wireless Communications. Artech House, 2012.

[4] Y Cho and H Yoo. Miniaturised dual-band implantable antenna for wireless biotelemetry. Electronics Letters, 52(12):1005-1007, 2016.

[5] Xiong Ying Liu, Ze Tao Wu, Yi Fan, and Emmanouil M Tentzeris. A miniaturized csrr loaded wide-beamwidth circularly polarized implantable antenna for subcutaneous real-time glucose monitoring IEEE Antennas and Wireless Propagation Letters, 16:577-580, 2017.

[6] Tuba Yilmaz, Robert Foster, and Yang Hao. Broadband tissue mimicking phantoms and a patch resonator for evaluating noninvasive monitoring of blood glucose levels. IEEE Transactions on Antennas and Propagation, 62(6):3064-3075, 2014.

[7] Hua Li, Yong-Xin Guo, Changrong Liu, Shaoqiu Xiao, and Lin Li. A miniature-implantable antenna for medradio-band biomedical telemetry. IEEE Antennas and Wireless Propagation Letters, 14:11761179, 2015.

[8] S. Gabriel, R. W. Lau, and C. Gabriel. The dielectric properties of biological tissues: II. measurements in the frequency range $10 \mathrm{~Hz}$ to 20 GHz. Physics in Medicine and Biology, 41(11):2251-2269, 1996. 